\title{
Expression of Lewis y antigen and integrin av, $\beta 3$ in ovarian cancer and their relationship with chemotherapeutic drug resistance
}

\author{
Jian Gao, Zhenhua Hu, Dawo Liu, Juanjuan Liu, Chuan Liu, Rui Hou, Song Gao, Danye Zhang, Shulan Zhang \\ and Bei Lin ${ }^{*}$
}

\begin{abstract}
Objective: This study investigates the expression of Lewis y antigen, integrin av, $\beta 3$ in epithelial ovarian cancer tissues. We further evaluate the relationship between their expression and chemotherapy resistance of ovarian cancer and its possible clinical significance.

Methods: Tissues of 92 patients with ovarian cancer meeting the inclusion criteria with complete follow-up data were enrolled and divided into chemotherapy resistant group and sensitive group. The expression and relationship of Lewis y antigen and integrin av, $\beta 3$ are assessed in paraffin sections using immunohistochemistry and doublelabeling immunofluorescence method. Multivariate logistic regression analysis was used to investigate the relationship between age, clinical stage, differentiation, histologic subtype, Lewis y antigen and integrin av, $\beta 3$ expression in ovarian cancer patients.
\end{abstract}

Results: The expression rates of Lewis y antigen and integrin av in the resistant group, significantly higher than the rates found in the sensitive group $(p<0.05)$. Multivariate analysis showed that the expression of Lewis $y$ antigen, integrin av and ovarian cancer's clinical stage were independent, drug resistance-related risk factors. The expression levels of Lewis y antigen and integrin av, $\beta 3$ were positively correlated with each other.

Conclusions: A close correlation between Lewis y antigen, integrin $a v, \beta 3$ and ovarian cancer was observed. Lewis $y$ antigen can influence the biological behavior of a tumor cell as an important composition of integrin av, $\beta 3$ by some signal pathway. And the expression of Lewis y antigen, integrin av and ovarian cancer's clinical stage are both independent, drug resistance-related risk factors.

Keywords: Ovarian Cancer, Lewis y Antigen, Integrin av, $\beta 3$, Chemotherapeutic Drug Resistance

\section{Background}

Ovarian cancer has the highest mortality rate of all cancers of the female reproductive system. Chemotherapy resistance is an important factor influencing treatment efficacy. In recent years, studies have shown that through the interaction between surface adhesion molecules and surrounding extracellular matrix, tumor cells can promote proliferation, invasion, and metastasis, thus improving their tolerance to chemotherapeutic drugs [1]. Cell adhesion-mediated drug resistance (CAM-DR) is a relatively new theory for the mechanism of drug

\footnotetext{
* Correspondence: linbei88@hotmail.com

Department of Obstetrics and Gynecology, Shengjing Hospital Affiliated to China Medical University, Shenyang 110004, China
}

resistance in tumor cells [2-4]. Integrins comprise a large family of cell adhesion molecules; they are transmembrane glycoproteins that play a key role in the interaction between cells and components of the interstitial space. Integrin-mediated interactions between cells or between cells and the extracellular matrix play an important role in tumor growth, invasion, metastasis, drug resistance, and many other processes [5]. Many studies have confirmed that carbohydrate antigens on the cell surface are closely related to integrins. In our previous work, we have found that as a part of the integrin $\alpha v \beta 3$ structure, Lewis y antigen expression is related to the degree of invasiveness of ovarian cancer [6]. Here we use immunohistochemistry to further study the expression 
of Lewis y antigen and integrin $\alpha v \beta 3$ in tissue specimens from patients with chemotherapy resistant or sensitive ovarian cancer and analyze how the expression of these molecules correlates with chemotherapy resistance and the resulting clinical significance.

\section{Materials and methods}

92 chosen paraffin samples are obtained from the operations done from 2006 to 2010 in the department of Gynecology and Obstetrics of Sheng Jing Hospital Affiliated to China Medical University. After the cytoreductive surgery and 6-8 periods of systematic chemotherapy, each patient will receive a follow up observation for at least one year. Among the 92 cases of primary epithelial ovarian cancer studied, there are 58 cases of serous cystadenocarcinoma, 8 mucinous cystadenocarcinoma, 4 endometrioid carcinoma, 7 clear cell carcinoma and 15 poorly differentiated adenocarcinoma. According to histological grade, there were 15 cases of high differentiated, 35 moderate and 42 poor. The group includes 19 cases of stages I, 13 stages II, and 60 stages III (according to International Federation of Gynecology and Obstetrics (FIGO) criteria). All the cases are primary, the information and follow-up data are complete; chemical treatment is not used in all the patients before operations.

\section{Drug resistance related clinical and pathological parameters}

Tissues obtained between 2006 and 2010 from 92 patients with ovarian cancer meeting the inclusion criteria with complete follow-up data were enrolled. The clinical and pathological parameters of ovarian cancer patients include age, clinical stage, differentiation, histologic subtype and chemotherapy scheme (PTX (paclitaxel) + Carboplatin (TC)). According to the guideline of National Comprehensive Cancer Network (NCCN) (recurrence during the chemotherapy period or within 6 months after the chemotherapy was define as drug resistance group; after the chemotherapy recurrence between 6 to 12 months was partial sensitive group and recurrence beyond 12 months after the chemotherapy or didn't recurrenc was sensitive group), the patients were divided into chemotherapy resistant group (34 cases) and sensitive group (58 cases).

\section{Main reagents}

Mouse monoclonal anti-Lewis y antibody (clone A 70$\mathrm{C} / \mathrm{C} 8$ ) was purchased from Abcam Company (UK). Rabbit polyclonal anti- $\alpha v$ and anti- $\beta 3$ antibodies were purchased from Santa Cruz (USA). Goat monoclonal anti-rabbit immunoglobulin $\mathrm{G}$ fluorescein isothiocyanate (FITC) and goat monoclonal anti-mouse immunoglobulin $\mathrm{G}$ tetramethyl rhodamine isothiocyanate (TRITC) were purchased from Fujian Maixin Company (China).
DAPI was purchased from Shenyang Baoxin Company (China). Serum albumin (BSA) and DAB kit were purchased from Zhongshan Biotechnology Company (China). Other reagents were supplied by our laboratory.

\section{Methods}

Immunohistochemistry

Streptavidin-biotin-peroxidase (SP) immunohistochemistry was performed. Tissues were fixed in $4 \%$ formaldehyde and embedded in paraffin, and $4 \mathrm{~mm}$ thick serial sections were prepared at the same organizational part. The working dilution of Lewis y antibody and integrin $\alpha v, \beta 3$ antibody were $1: 100$ and $1: 160$, respectively. The staining procedure was performed according to SP kit manual. The group with PBS instead of primary antibody was used as a negative control. A colon cancer sample served as positive control for Lewis y antigen, and a breast cancer sample was a positive control for integrin $\alpha \mathrm{v}, \beta 3$.

\section{Immunofluorescence}

The sample slices of strong expression for immunohistochemistry were selected to performed immunofluorescence double labeling method. Primary antibody combinations were anti-integrin $\alpha v$ with anti-Lewis y, or anti-integrin $\beta 3$ with anti-Lewis $y$, with the PBS instead of primary antibody as the negative control. The working dilution of rabbit anti-human integrin $\alpha v, \beta 3$ and mouse anti-human Lewis $y$ antibody were all 1:160. The working dilution of goat antirabbit IgM FITC and goat anti-mouse IgG TRITC were 1:100. The working dilution of nuclear dye DAPI was 1:100. The staining was performed according to the instructions of immunofluorescence kit.

\section{The determination of results}

The presence of brown colored granules on the cell membrane or in the cytoplasm was taken as a positive signal, and was divided by color intensity into not colored, light yellow, brown, tan and was recorded as 0 , 1,2 , and 3 , respectively. We choose five high-power fields in series from each slice, then score them and take the average percentage of chromatosis cells. A positive cell rate of less than $5 \%$ was $0,5 \sim 25 \%$ was $1,26 \sim 50 \%$ was 2 , $51 \sim 75 \%$ was 3 , more than $75 \%$ was 4 . The final score was determined by multiplying positive cell rate and score values: $0 \sim 2$ was considered negative $(-), 3 \sim$ 4 was $(+), 5 \sim 8$ was $(++), 9 \sim 12$ was $(+++)$. The results were read by two independent observers to control for variability.

Microscopic red fluorescence indicated Lewis y antigen labeled by TRITC, green fluorescence indicated integrin $\alpha v, \beta 3$ labeled by FITC, while blue fluorescence indicated DAPI-stained nucleus. Pictures of the three individual fluorescence channels were superimposed 
using image analysis software, with a yellow fluorescence indicated co-localization of Lewis y antigen and integrin $\alpha v, \beta 3$.

\section{Statistical analysis}

Statistical analyses were performed using the SPSS software Version 11.5. Data expressed as mean \pm SD was applied for statistical analysis. The Student's $t$ test was applied to compare data between the two groups, and analysis of variance was applied to compare data among multiple groups.

The Chi-square $(x 2)$ test was applied to analyze the expression of Lewis y antigen, integrin $\alpha v, \beta 3$ and clinicopathological parameters. The Spearman correlation analysis method was applied to calculate the coefficient $R$ of indexes and to analyze its correlation, A $P$ value $<0.05$ was considered statistically significant.

\section{Results}

Expression of Lewis y antigen, integrin av and $\beta 3$ in different groups

Lewis y antigen was expressed in the cytoplasm and cell membrane, mainly on membrane and rarely in the nucleus. The expression rates of Lewis y antigen in the resistant group were $91.67 \%$, significantly higher than $60.34 \%$ in the sensitive group $(p<0.05)$, as shown in Figure 1 and Table 1.

Similar to Lewis $y$, the expression of integrin $\alpha v$ and $\beta 3$ were mainly on membrane. The integrin $\alpha v$ positive expression rate was $85.29 \%$ in the resistant group, significantly higher than that of the sensitive group (51.72\%) $(P<0.05)$. The expression rate of integrin $\beta 3$ in the resistant group was $88.24 \%$, higher than $65.52 \%$ in the sensitive group, but there were no significant difference between these two groups $(p>0.05)$, Figure 1 and Table 2 .

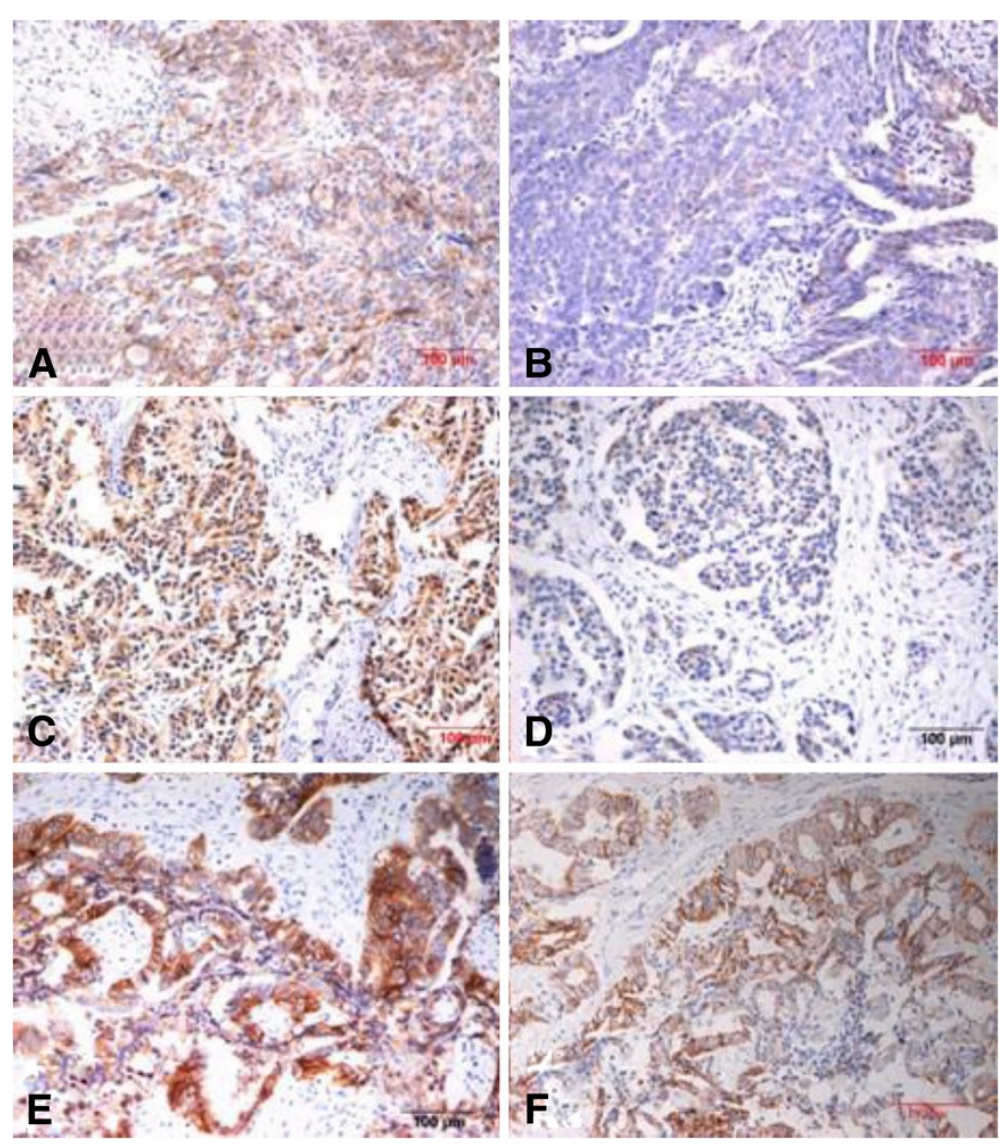

Figure 1 Expression of Lewis y antigen in resistant group (Fig.A: stage Illc, moderate differentiated serous cystadenocarcinoma) and sensitive group (Fig.B: stage IIlc, poorly differentiated serous cystadenocarcinoma)(*200); Expression of integrin av in resistant group (Fig.C: stage IIIc, moderate differentiated serous cystadenocarcinoma) and sensitive group (Fig.D: stage Illc, moderate differentiated serous cystadenocarcinoma)(*200); Expression of Lewis y antigen in resistant group (Fig.E: stage IIlc, moderate differentiated endometrioid carcinoma)and sensitive group (Fig.F: stage Illc, moderate differentiated endometrioid carcinoma)(*200). 
Table 1 Expression of Lewis $y$ antigen in different groups

\begin{tabular}{|c|c|c|c|c|c|c|c|}
\hline \multirow[t]{2}{*}{ Groups } & \multirow[t]{2}{*}{ Cases } & \multicolumn{4}{|c|}{ Lewis y antigen } & \multirow{2}{*}{$\begin{array}{l}\text { Positive } \\
\text { cases }\end{array}$} & \multirow{2}{*}{$\begin{array}{l}\text { Positive } \\
\text { rate (\%) }\end{array}$} \\
\hline & & - & + & ++ & $\overline{+++}$ & & \\
\hline Resistant group & 34 & 3 & 4 & 19 & 8 & 31 & 91.18 \\
\hline Sensitive group & 58 & 23 & 16 & 19 & 0 & 36 & 60.34 \\
\hline
\end{tabular}

\section{Drug resistance-related risk factors univariate analysis}

The clinical and pathological parameters of ovarian cancer patients include age, clinical stage, differentiation, histologic subtype, only ovarian cancer's clinical stage were independent, drug resistance-related risk factors $(P=0.01)$, the difference between the rest factors was not significant $(p>0.05)$, as shown in Table 3 .

\section{Drug resistance-related risk factors multivariate logistic regression analysis}

Multivariate logistic regression analysis was used to investigate the relationship between age, clinical stage, differentiation, histologic subtype, and Lewis y antigen and integrin $\alpha v \beta 3$ expression in ovarian cancer patients with ovarian cancer chemotherapy resistance. The result showed that both the expression of Lewis $y$ antigen and integrin $\alpha \mathrm{v}$ and ovarian cancer's clinical stage were independent, drug resistance-related risk factors, as shown in Table 4.

In addition, immunofluorescence double-labeling revealed that in ovarian cancer Lewis y antigen (red fluorescence) was localized in the cell membrane and cytoplasm. Integrin $\alpha \mathrm{v}$ and $\beta 3$ (green fluorescence) were mainly localized in the cell membrane, with a small amount of coloring in the cytoplasm. The 4,6-diamino-2-phenyl indole (DAPI) (blue fluorescence) was used to visualize the nucleus. In three-channel synthesized images, the yellow fluorescence emerges from the area emitting both red and green fluorescence, indicating co-localization of Lewis y antigen and integrin $\alpha v, \beta 3$, as shown in Figure 2.

\section{Correlation analysis between expression of Lewis $y$ antigen and integrin av, $\beta 3$ in ovarian cancer}

A similar trend was seen in the expression of Lewis $y$ antigen, integrin $\alpha \mathrm{v}, \beta 3$ in 92 patients with ovarian cancer, according to the results of immunohistochemistry. Both Lewis y antigen and integrin $\alpha v \beta 3$ showed high expression in the ovarian cancer resistant group and their expression levels were positively correlated with each other (Tables 5, 6 Figure 1).

In order to investigate the correlation between these two antigens, we performed Spearman correlation analysis for each set of data, which demonstrated a high degree of positive correlation between Lewis y and integrin $\alpha v$ (correlation coefficients were $0.235, P<0.05$ ), $\beta 3$ (correlation coefficients were $0.333, P<0.01$ ) subunits.

\section{Discussion}

Chemotherapy resistance has been proven to be a very difficult issue in the treatment of ovarian cancer. The mechanisms of resistance and appropriate countermeasures targeting these mechanisms have become hotspots in ovarian cancer research. Previous studies of the mechanism of resistance in ovarian cancer mainly focused on drug concentration in tumor cells, DNA damage repair mechanisms, glutathione-dependent detoxification enzyme system activity, and other aspects. In recent years, a number of studies on malignant tumor drug resistance have found that tumor drug resistance is related to changes in adhesion molecule composition, the adhesion abilities of tumor cells, and the resultant cytoskeletal rearrangements and signal transduction pathway activation. Therefore, a new mechanism of tumor drug resistance-cell adhesion mediated drug resistance (CAM-DR) has been proposed [2-4].

The adhesion of tumor cells to the surrounding environment can improve cell survival and anti-apoptotic ability. Integrins are important cell surface adhesion molecules as they are receptors for many extracellular matrix components. Integrin receptors can regulate cell growth, differentiation, and metastasis through transmembrane signal transduction. Tumor cell growth and metastasis are both closely related to drug resistance. Metastasized tumor cells are more likely to be drug resistant and resistant tumor cells have a stronger ability to metastasize or invade. The relationship between integrins and drug resistance is gradually gaining recognition, but the research is still in early stages [7-9]. Damiano et al [10] found that the expression of integrin $\alpha 4 \beta 1$ in the drug-resistant strain, RPMI8226/S, of human multiple myeloma cell strain RPMI8226 was significantly higher than that in sensitive strains; furthermore, extracellular matrix-coated cells significantly increased the cells' tolerance of the chemotherapeutic drugs

Table 2 Expression of integrin $\alpha v$ and $\beta 3$ in different groups

\begin{tabular}{|c|c|c|c|c|c|c|c|c|c|c|c|c|c|}
\hline \multirow[t]{2}{*}{ Groups } & \multirow[t]{2}{*}{ Cases } & \multicolumn{6}{|c|}{ Integrin av } & \multicolumn{6}{|c|}{ Integrin $\beta 3$} \\
\hline & & - & + & ++ & +++ & Positive cases & Positive rate(\%) & - & + & ++ & +++ & Positive cases & Positive rate(\%) \\
\hline Resistant group & 34 & 5 & 8 & 11 & 10 & 29 & 85.29 & 4 & 10 & 10 & 10 & 30 & 88.24 \\
\hline Sensitive group & 58 & 28 & 16 & 6 & 8 & 30 & 51.72 & 20 & 21 & 15 & 2 & 38 & 65.52 \\
\hline
\end{tabular}


Table 3 Drug resistance-related risk factors univariate analysis

\begin{tabular}{|c|c|c|c|c|c|c|}
\hline \multirow[t]{2}{*}{ 分组 } & \multirow{2}{*}{$\begin{array}{l}\text { Total } \\
\text { cases }\end{array}$} & \multicolumn{2}{|c|}{$\begin{array}{l}\text { Resistant } \\
\text { group }\end{array}$} & \multicolumn{2}{|c|}{$\begin{array}{l}\text { Sensitive } \\
\text { group }\end{array}$} & \multirow[t]{2}{*}{$P$} \\
\hline & & Cases & $\begin{array}{c}\text { Rate } \\
(\%)\end{array}$ & Cases & $\begin{array}{c}\text { Rate } \\
(\%)\end{array}$ & \\
\hline \multicolumn{7}{|l|}{ FIGO Stages } \\
\hline । & 19 & 1 & 2.94 & 18 & 31.03 & 0.01 \\
\hline$\|$ & 13 & 3 & 8.82 & 10 & 17.24 & \\
\hline III & 60 & 30 & 88.24 & 30 & 51.72 & \\
\hline IV & 0 & 0 & 0 & 0 & 0 & \\
\hline \multicolumn{7}{|l|}{ Differentiation } \\
\hline High & 15 & 5 & 14.71 & 10 & 17.24 & 0.298 \\
\hline Moderate & 35 & 12 & 35.29 & 23 & 39.66 & \\
\hline Poorly & 42 & 17 & 50.00 & 25 & 43.10 & \\
\hline \multicolumn{7}{|l|}{ Histologic subtype } \\
\hline $\begin{array}{l}\text { Serous } \\
\text { cystadenocarcinoma }\end{array}$ & 58 & 24 & 70.59 & 34 & 58.62 & 0.872 \\
\hline $\begin{array}{l}\text { Mucinous } \\
\text { cystadenocarcinoma }\end{array}$ & 8 & 4 & 11.76 & 4 & 6.90 & \\
\hline Endometrioid carcinoma & 4 & 1 & 2.94 & 3 & 5.17 & \\
\hline Clear cell carcinoma & 7 & 1 & 2.94 & 6 & 10.34 & \\
\hline $\begin{array}{l}\text { Poorly differentiated } \\
\text { adenocarcinoma }\end{array}$ & 15 & 4 & 11.76 & 11 & 18.87 & \\
\hline
\end{tabular}

melphalan and doxorubicin and reduced the rate of apoptosis. Similar findings have been observed for leukemia, glioma, breast cancer and small cell lung cancer. In preliminary studies, we have also demonstrated that the ovarian cancer cell line, RMG-I-h, with high expression of the integrins $\alpha 5 \beta 1$ and $\alpha v \beta 3$, can increase drug resistance to 5-FU, carboplatin, and paclitaxel $[11,12]$.

Integrin glycosylation status has been shown to affect the strength of integrin-ligand binding and the formation of the glycosidic bond catalyzed by glycosyltransferase affecting the glycosylation status of integrins. The fucose sugar has been shown to be a component of integrin molecules. Furthermore, core fucosylation is

Table 4 Drug resistance-related risk factors multivariate logistic regression analysis

\begin{tabular}{clllll}
\hline Factors & $\mathbf{B}$ & $\mathbf{S x}$ & $\mathbf{P}$ & $\mathbf{O R}$ & $\mathbf{9 5 \%} \mathbf{C l}$ \\
\hline Lewis y antigen & -2.249 & 0.605 & 0.000 & 0.106 & 0.0320 .345 \\
Integrin av & -0.968 & 0.415 & 0.020 & 0.380 & 0.1680 .857 \\
Clinical stage & -1.304 & 0.575 & 0.023 & 0.271 & 0.0880 .838 \\
\hline
\end{tabular}

$B$ : model parameter.

Sx: standard error of mean.

$P$ : $P$ value.

$O R$ : odds ratio. essential for integrin-mediated cell migration and signal transduction and plays a key role in the interaction between cells and extracellular matrix, thus affecting tumor metastasis. E. W. Easton et al [13] purified $\alpha 5 \beta 1$ integrin from human placenta and $\alpha 3 \beta 1$ integrin from the uterine epithelial cell line, HCV29, and demonstrated that both integrins were more than 50\% fucosylated. Zhao et al [14] found that knockout of the $\alpha 1,6$-fucose transferase gene (FT8) could prevent integrin $\alpha 3 \beta 1$-mediated cell migration and cell growth signals, suggesting that core fucosylation is required for the functions of integrin $\alpha 3 \beta 1$. Lewis $y$ antigen is an oligosaccharide containing two fucose molecules and falls into the A, B, H, and Lewis blood type families. The role of Lewis y antigen as a cancer-associated antigen in tumorigenesis and development gradually arouses more concern. We have previously demonstrated that the Lewis $y$ antigen is a part of the $\alpha 5 \beta 1$ and $\alpha v \beta 3$ structures and high expression of Lewis y antigen and integrins $\alpha 5 \beta 1$ and $\alpha v \beta 3$ can enhance the proliferative and adhesive abilities of cells $[6,15]$. Furthermore, we have shown We have also previously shown that cell lines and clinical ovarian cancer specimens exhibiting increased expression of Lewis y antigens in integrins $\alpha 5 \beta 1$ and $\alpha v \beta 3$ are more likely to exhibit a malignant phenotype $[6,15,16]$. Our studies have also shown that Lewis y antigen can increase the ability of $\alpha 5 \beta 1$ and $\alpha v \beta 3$ to bind their ligands, fibronectin (FN) and vitronectin (VN), thereby increasing the cells' resistance to platinum drugs by enhancing cellular adhesion $[6,15,17]$.

On the basis of this body of work, we retrospectively analyzed the expression of Lewis y antigen and integrin $\alpha v \beta 3$ in the tissue specimens of patients resistant to platinum drugs and investigated their relationship with drug resistance. We found the rates of expression of Lewis $y$ antigen and $\alpha v$ integrins in the resistant group were significantly higher than those in the sensitive group $(P<0.05)$; however, the expression rate of integrin $\beta 3$ in the two groups was not significantly different. Multivariate analysis showed that the expression of Lewis $y$-antigen and integrin $\alpha v$ and the clinical stage of ovarian cancer were both independent drug resistancerelated risk factors, suggesting that the detection of Lewis y antigen and integrin $\alpha v \beta 3$ could play an important role in the prediction of ovarian cancer patients' drug resistance, prognosis, and outcome. Correlation analysis showed that Lewis y antigen and integrin subunits $\alpha v$ and $\beta 3$ in ovarian cancer tissues were highly expressed in ovarian cancer cells and their expression levels were positively correlated with each other. Dualcolor immunofluorescence labeling indicated that Lewis $y$ antigen and integrin $\alpha v \beta 3$ were co-localized in ovarian cancer tissues, further confirming their correlation of expression. 


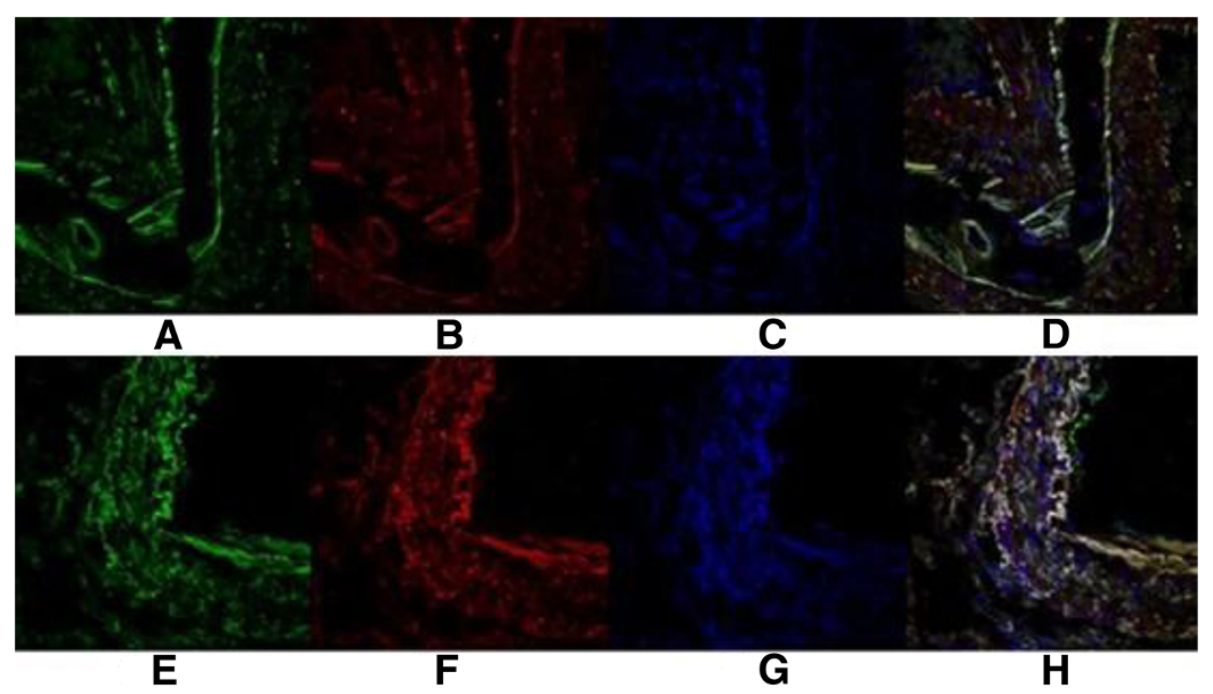

Figure 2 Integrin $\mathbf{a v}, \boldsymbol{\beta} 3$ and Lewis $\mathbf{y}$ colocalize in ovarian malignant tumor. Integrin av and $\beta 3(\mathbf{A}$ and $\mathbf{E}) ;$ Lewis y (B and $\mathbf{F}) ;$ Nucleus (C and $\mathbf{G})$; Merged image (D and $\mathbf{H})(* 400)$.

The adhesion between cells and extracellular matrix can increase the activity of protein tyrosine kinase (PTK), resulting in more tumor cells in G1 phase and phosphoinositide 3 kinase activation [18]. Lewis y antigen is not only a part of the integrin $\alpha 5 \beta 1$ and $\alpha v \beta 3$ structures, but is also a part of the structure of other adhesion molecules such as CD44 [19]. Therefore, increased expression of Lewis $y$ antigen can improve the adhesion of cells to the matrix and promote cell adhesion and metastasis through corresponding signal transduction pathways. These actions can then enhance cell behaviors that promote malignancy which provides a theoretical basis for altering Lewis y antigen expression and/or downstream signaling modification in the treatment of ovarian cancer. Although the mechanism by which adhesion molecule fucosylation affects drug resistance is not yet clear, it is currently believed that integrin-mediated tumor cell resistance is related to the following factors: (1) regulating apoptosis (Bax/BclX); (2) changing the drug targets (of Topo II); (3) inhibiting
DNA injury, and enhancing DNA repair; (4) regulating P27 protein, etc. Our studies have shown that Lewis yantigen is involved in the aforementioned process, and increases tumor cell drug resistance $[15,17]$. As a part of the integrin $\alpha 5 \beta 1$ and $\alpha v \beta 3$ structures, Lewis $y$ antigen can promote the adhesion of integrins to extracellular matrix in order to strengthen focal adhesion kinase (FAK) phosphorylation; increased expression of Lewis y antigen would activate FAK signal transduction pathways, increase cell adhesion, and increase drug resistance by regulating Topo-T, Topo-Ii $\beta$, Bcl-2, and Bcl-XL.

These results suggest that the immunohistochemical detection of Lewis $y$ antigen and integrin $\alpha v \beta 3$ in ovarian cancer tissues can be used as important indicators for determining appropriate clinical chemotherapy, prognosis, and outcome. In-depth understanding of signaling transduction pathways for integrinmediated chemotherapy resistance will provide a basis for increasing chemosensitivity and developing new chemotherapies.
Table 5 Correlation between expression of Lewis y antigen and integrin av in ovarian cancer

\begin{tabular}{cccc}
\hline \multirow{2}{*}{$\begin{array}{c}\text { Lewis } \mathbf{y} \\
\text { antigen }\end{array}$} & \multicolumn{2}{c}{ Integrin av } & Total \\
\cline { 2 - 3 } & Positive cases & Negative cases & \\
\hline Positive cases & 46 & 20 & 66 \\
Negative cases & 13 & 13 & 26 \\
Total & 59 & 33 & 92 \\
\hline
\end{tabular}

Table 6 Correlation between expression of Lewis y antigen and integrin $\beta 3$ in ovarian cancer

\begin{tabular}{cccc}
\hline \multirow{2}{*}{$\begin{array}{l}\text { Lewis } \boldsymbol{y} \\
\text { antigen }\end{array}$} & \multicolumn{2}{c}{ Integrin $\boldsymbol{\beta 3}$} & Total \\
\cline { 2 - 3 } & Positive cases & Negative cases & \\
\hline Positive cases & 52 & 14 & 66 \\
Negative cases & 16 & 10 & 26 \\
Total & 68 & 24 & 92 \\
\hline
\end{tabular}




\section{Competing interests}

The authors declare that they have no competing interests.

\section{Authors' contributions}

JG carried out most parts of the experiment; $\mathrm{CL}, \mathrm{RH}, \mathrm{SG}$ and $\mathrm{DZ}$ participated in the experiment; $\mathrm{BL}$ and $\mathrm{SZ}$ participated in the design of the study; $\mathrm{DL}$ and $J$ performed the statistical analysis; ZH participated in its design and coordination and helped to draft the manuscript. All authors read and approved the final manuscript.

\section{Acknowledgements}

This work was supported by the National Natural Science Foundation of China $(30571985,30872757,81072118)$.

Received: 13 December 2012 Accepted: 16 January 2013

Published: 1 June 2013

\section{References}

1. Skubitz AP: Adhesion molecules. Cancer Treat Res 2002, 107:305-329.

2. Hazlehurst LA, Dalton WS: Mechanisms associated with cell adhesion mediated drug resistance (CAM-DR) in hematopoietic malignancies. Cancer Metastasis Rev 2001, 20:43-50.

3. Damiano JS: Integrins as novel drug targets for overcoming innate drug resistance. Curr Cancer Drug Targets 2002, 2:37-43.

4. Moro L, Venturino M, Bozzo C, Silenqo L, Altruda F, Bequinot L, et al: Integrins induce activation of EGF receptor: role in MAP kinase induction and adhesion-dependent cell survival. EMBO J 1998, 17:6622-6632.

5. NikoloPoulos SN, Blaikie P, Yoshioka T, Guo W, Giancotti FG: Integrin beta4 signaling promotes tumor angiogenesis. Cancer Cell 2004, 6:471-483.

6. Wang Y, Liu J, Lin B, Wang C, Li Q, Liu S, et al: Study on the expression and clinical significances of Lewis y antigen and integrin av, $\beta 3$ in epithelial ovarian tumors. Int J Mol Sci 2011, 12:3409-3421.

7. Taylor ST, Hickman JA, Dive C: Epigenetic determinants of resistance to etoposide regulation of $\mathrm{BCl}-\mathrm{X}(\mathrm{L})$ and Bax by tumor microenvironmental factors. J Natl Cancer Inst 2000, 92:18-23.

8. Li J, Yen C, Liaw D, Podsypanina K, Bose S, Wang Sl, et al: PTEN, a putative protein tyrosine phosphatase gene mutated in human brain, breast, and prostate cancer. Science 1997, 275:1943-1947.

9. Steck PA, Perhouse MA, Jasser SA, Yung WK, Lin H, Ligon AH, et al: Identification of a candidate tumour suppressor gene, MMAC1, at chromosome 10q23. 3 that is mutated in multiple advanced cancer. Nat Genet 1997, 15:356-362.

10. Damiano JS, Cress AE, Hazlehurst LA, Shtil AA, Dalton WS: Cell adhesion mediated drug resistance (CAM-DR): role of integrins and resistance to apoptosis in human myeloma cell lines. Blood 1999, 93:1658-1667.

11. Zhang F, Liu J, Lin B, Liu Q, Zhao Y, Zhu L, et al: Increase in docetaxelresistance of ovarian carcinoma-derived RMG-1 cells with enhanced expression of Lewis Y antigen. Int J Mol Sci 2011, 12:7323-7334.

12. Iwamori M, Tanaka K, Kubushiro K, Lin B, Kiquchi K, Ishiwata I, et al: Alterations in the glycolipid composition and cellular properties of ovarian carcinoma-derived RMG-1 cells on transfection of the a1, 2-fucosyltransferase gene. Cancer Sci 2005, 96(1):26-30.

13. Easton EW, Bolsche JG, van den Eijnden DH: Enzymatic amplification involving glycosyltransferases forms the basis for the increased size of asparagine-linked glycans at the surface of NIH 3 T3 cells expressing the N-ras proto-oncogene. J Boil Chem 1991, 266:21674-21680.

14. Zhao $Y$, Itoh S, Wang $X$, Miyoshi E, Kariya $Y$, Miyazaki $K$, et al: Deletion of core fucosylation on $a_{3} \beta_{1}$ integrin down-regulates its functions. $J$ Boil Chem 2006, 281(50):38343-38350.

15. Yan LM, Lin B, Zhu LC, Hao YY, Qi Y, Wang CZ, et al: Enhancement of the adhesive and spreading potentials of ovarian carcinoma RMG- 1 cells due to increased expression of integrin a5 $\beta 1$ with the LewisY-structure on transfection of the a1, 2-fucosyltransferase gene. Biochimie 2010, 92:852-857.

16. Li Q, Liu S, Lin B, Yan L, Wang Y, Wang C, et al: Expression and Correlation of Lewis y Antigen and Integrins a5 and $\beta 1$ in Ovarian Serous and Mucinous Carcinoma. Int J Gynecol Cancer 2010, 20:1482-1489.

17. Wang C, Yan L, Wang Y, Lin B, Liu S, Li Q, et al: Overexpression of Lewis (y) antigen protects ovarian cancer RMG-1 cells from carboplatin-induced apoptosis by the Upregulation of Topo-I and Topo-II b. The anatomical record 2011, 294:961-969.
18. Maubant S, Cruet-Hennequart S, Poulain L, Carreiras F, Sichel F, Luis J, et al: Altered adhesion properties and alphav integrin expression in a cisplatin-resistant human ovarian carcinoma cell line. Int J Cancer 2002, 97:186-194.

19. Gao L, Yan L, Lin B, Gao J, Liang X, Wang Y, et al: Enhancive effects of Lewis y antigen on CD44-mediated adhesion and spreading of human ovarian cancer cell line RMG-I. J Exp Clin Cancer Res 2011, 30(1):15.

doi:10.1186/1756-9966-32-36

Cite this article as: Gao et al:: Expression of Lewis y antigen and integrin $a v, \beta 3$ in ovarian cancer and their relationship with chemotherapeutic drug resistance. Journal of Experimental \& Clinical Cancer Research 2013 $32: 36$.

\section{Submit your next manuscript to BioMed Central and take full advantage of:}

- Convenient online submission

- Thorough peer review

- No space constraints or color figure charges

- Immediate publication on acceptance

- Inclusion in PubMed, CAS, Scopus and Google Scholar

- Research which is freely available for redistribution

Submit your manuscript at www.biomedcentral.com/submit
C Biomed Central 